\title{
Extensions to the recommended practices for GD\&T in STEP-AP210 in the context of packaged electronic components
}

\author{
Jamie Stori \\ SFM Technology, Inc. \\ Urbana, Illinois \\ Kevin Brady \\ National Institute of Standards and Technology \\ Electronics and Electrical Engineering Laboratory \\ Thomas Thurman \\ Rockwell Collins, \\ Cedar Rapids, Iowa
}

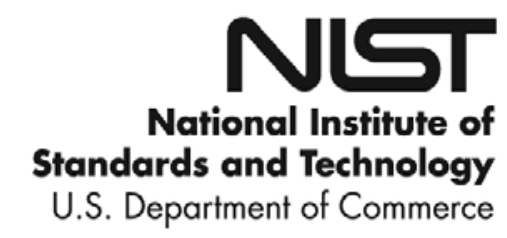


NISTIR 7634

\title{
Extensions to the recommended practices for GD\&T in STEP-AP210 in the context of packaged electronic components
}

\author{
Jamie Stori \\ SFM Technology, Inc. \\ Urbana, Illinois
}

Kevin Brady National Institute of Standards and Technology Electronics and Electrical Engineering Laboratory

Thomas Thurman Rockwell Collins, Cedar Rapids, Iowa

October 2009

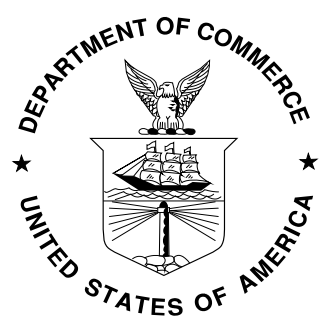

U.S. Department of Commerce Gary Locke, Secretary 


\title{
Extensions to the recommended practices for GD\&T in STEP-AP210 in the context of packaged electronic components
}

\author{
October 20, 2009
}

\section{Introduction}

The purpose of this document is to extend the recommended practices for the representation of GD\&T in STEP (ISO-10303) within the context of packaged electronic component models represented in AP210 (ISO-10303:210). The recommendations in this document are intended to be consistent with the current GD\&T accepted practice in STEP. Please refer to the document entitled "Recommended Practices for Dimensions, Dimensional and Geometric Tolerances” dated December 6, 2006 by David Briggs, Tom Hendrix, and Steve Yates for current practice recommendations for GD\&T in STEP. This document is available on the website of the CAx Implementor Forum (http://www.cax-if.org/) under the Recommended Practices link. The ARM application objects, relationships, attributes, and mapping to the corresponding MIM entities, relationships, and attributes have been updated to the IS version of the $2^{\text {nd }}$ Edition of AP210.

The largest family of standards for packaged electronic components are those published by JEDEC. The JEDEC standards are freely and publicly available, and contain detailed dimensioned and toleranced specifications for physical package configurations. JEDEC Publication 95 is a series of documents containing specifications for many common physical package configurations. The JEDEC Publication 95 documents can be accessed on-line at: http://www.jedec.org/DOWNLOAD/pub95/default.cfm. In this document, in addition to general guidelines for the representation of tolerances in a packaged electronic component, a set of STEP mappings for a typical toleranced JEDEC package model will be discussed. These examples are intended to be representative of many of the common tolerances encountered in the JEDEC standards.

The GD\&T standard referenced by both JEDEC and the 2006 STEP recommended practice is ASME Y14.5 (1994). The recommendations in this document are intended to be consistent with both the 1994 as well as recent 2009 publication of Y14.5.

\section{Notation}

ARM AOs will be denoted with a leading uppercase letter in Courier font (i.e. Datum) while a MIM entity will be displayed in all lowercase notation (i.e. datum).

\section{Assembly vs. Single-Solid representation of a packaged component model}

There are two primary alternatives for the representation of the three-dimensional geometric representation of a packaged component model. The preferred representation is one in which each of the physical package features (body, mounting features, terminals, etc.) have an explicit three-dimensional geometric model. In the case of a replicated 


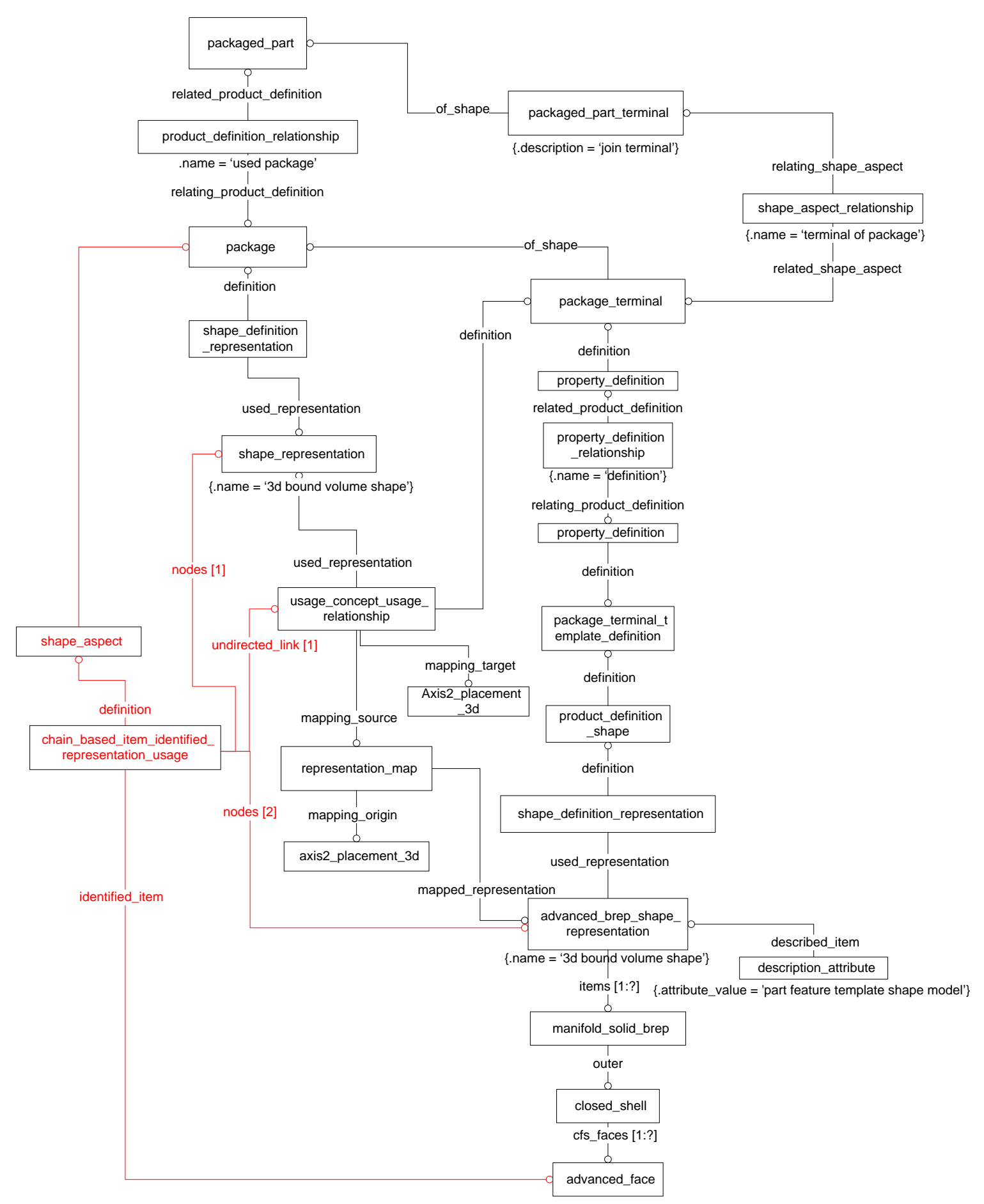

Figure 1. Defining a shape_aspect based on a surface of a terminal in a package model.

feature, such as a terminal, many terminal instances will share a common template (package_terminal_template_definition). Figure 1 through Figure 3 illustrate the preferred representation of the geometric model for a package_terminal, package_body, and seating_plane, respectively. As may be seen in the figures, each of the package features has a distinct shape_representation, typically an 


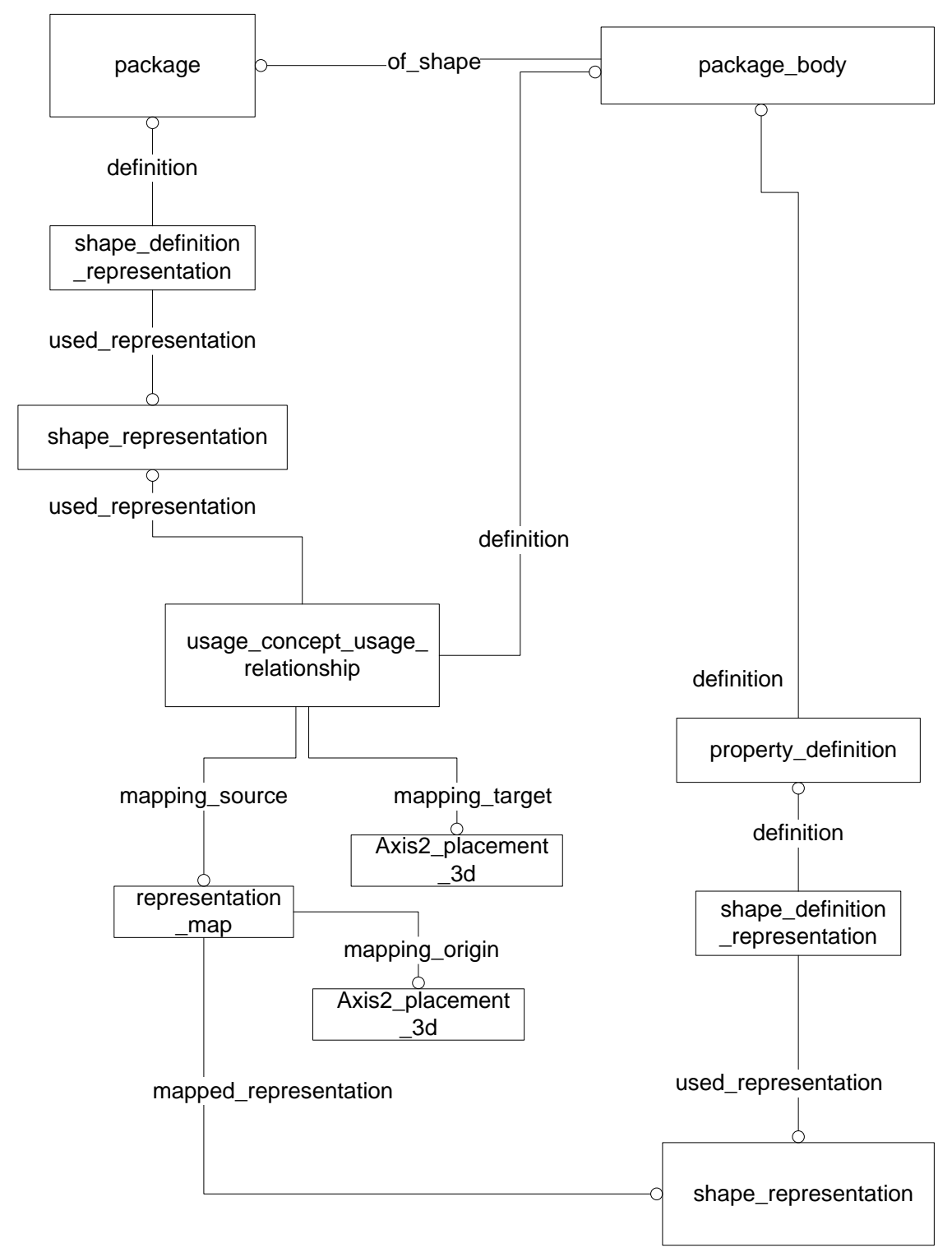

Figure 2. Relationship of the shape model of a package_body to that of the package.

advanced_brep_shape_representation. The package features are shape_aspects of the package, and the shape_representation of the feature is placed with respect to the shape_representation of the package with a usage_concept_usage_relationship. Explicit representation of the geometric models for distinct package features significantly enhances the ability to perform detailed domain-specific reasoning and functional analysis in contexts such as manufacturability, ECAD-MCAD integration, etc. This preferred representation is analogous to that a mechanical assembly, and the expression of tolerances and dimensions requires representation mechanisms similar to that of mechanical assemblies. 


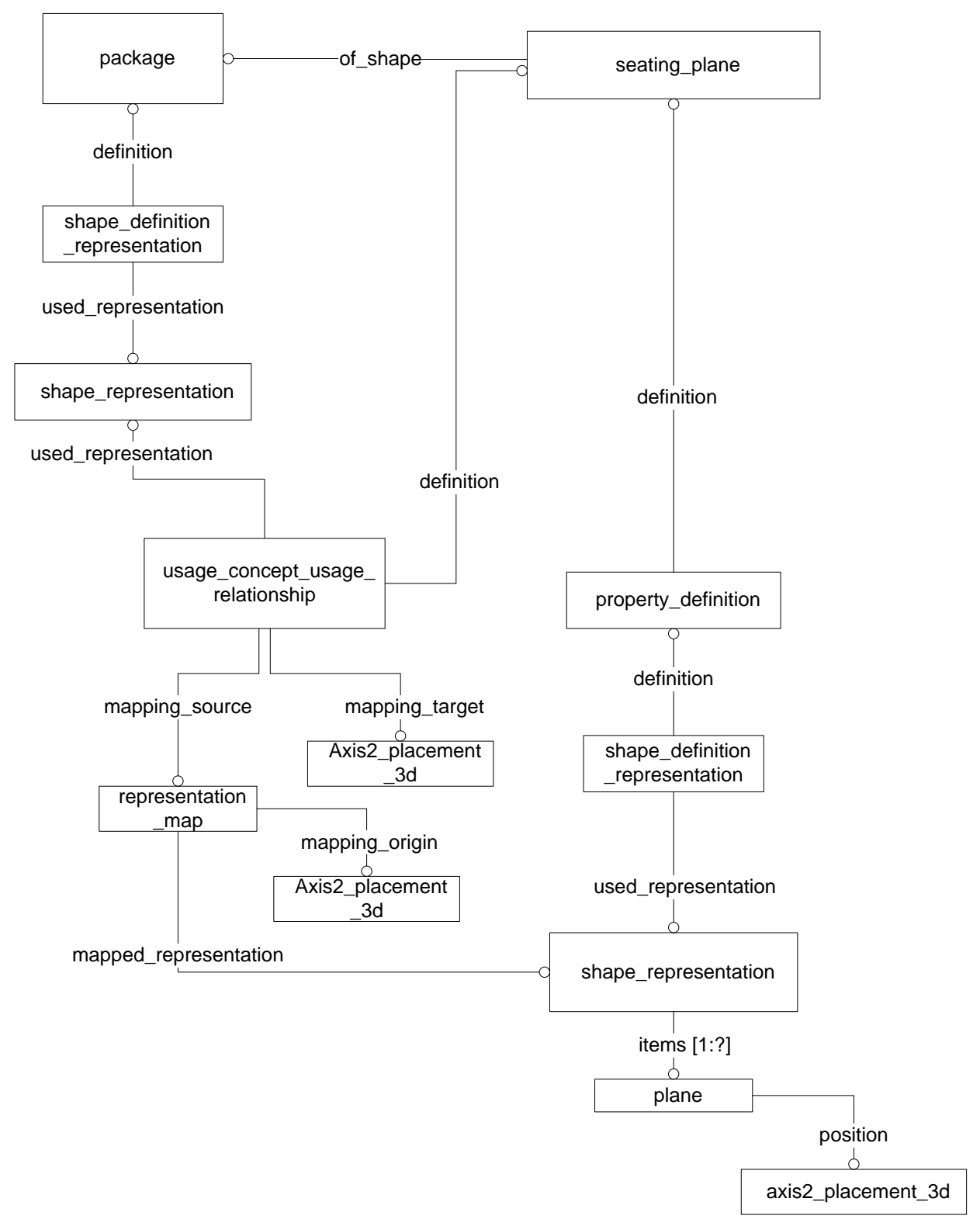

Figure 3. Representation of the seating_plane in a package model.

The alternative is to have a single shape_representation that contains the detailed geometric model for the package. In this case, dimensioning and tolerancing can be achieved through mechanisms applicable to a single-solid part.

\section{Shape_elements}

Features of the model relevant for GD\&T are represented by the Shape_element AO. Figure 4 shows several of the important subtypes of Shape_element. A surface of a part can be modeled directly as a Shape_element. In the MIM mapping, the feature will most likely be associated with an advanced_face. When it is desired to treat multiple disjoint regions as a single feature, a Composite_shape_element may be employed. A Composite_shape_element has two important subtypes - the 
Composite_group_shape_element and the Composite_unit_shape_element.A Composite_unit_shape_element is used to aggregate multiple Shape_elements that are to be treated as a unit. For example, multiple surfaces of a single feature-of-size.

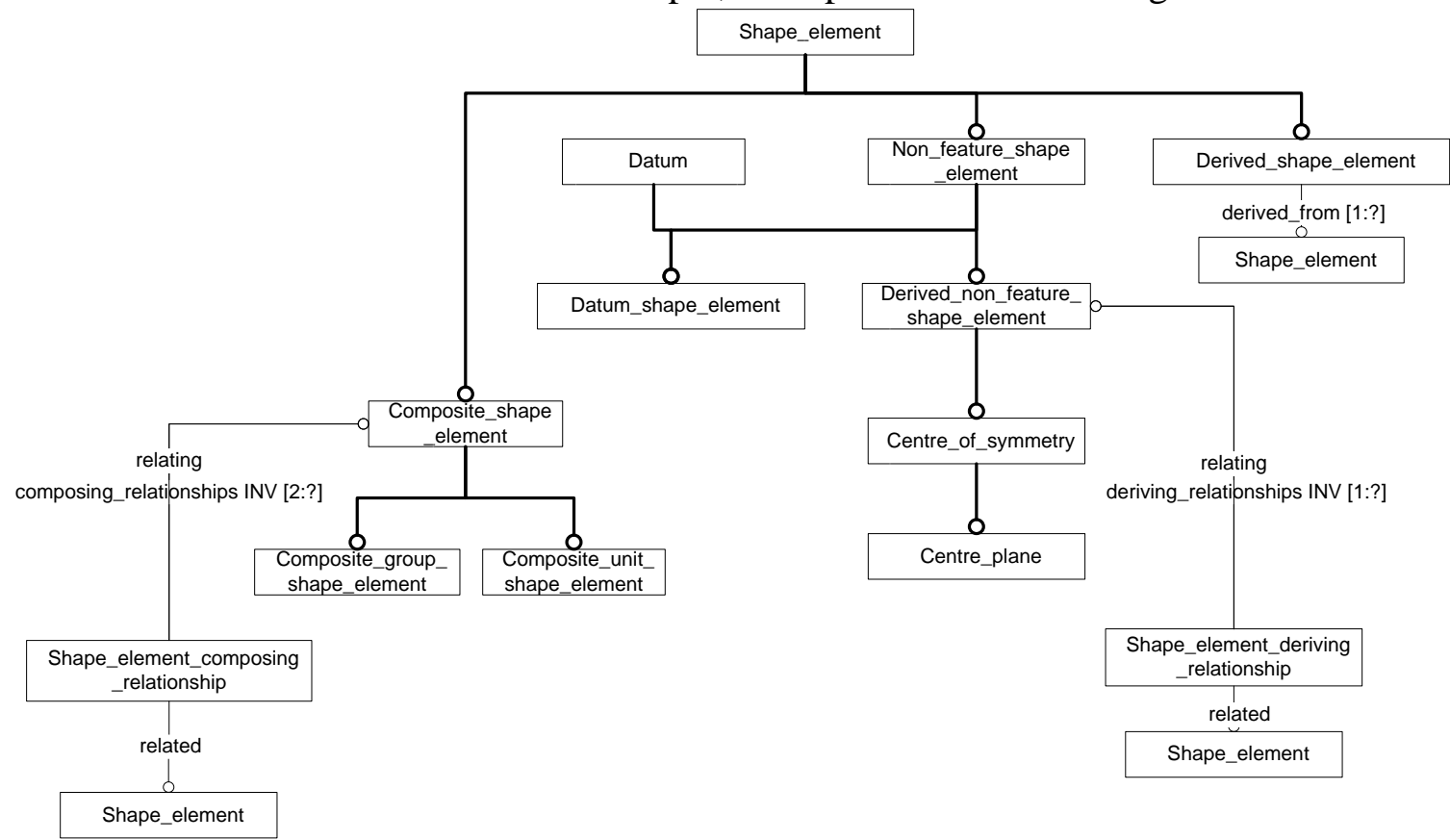

Figure 4. Several relevant subtypes of the Shape_element AO

A Composite_group_shape_element is used when it is desired to apply a property to each of the constituent elements individually. Figure 5 contains the MIM mapping of the Composite_shape_element (either composite_group_shape_aspect or composite_unit_shape_aspect) and the relationships to its constitutive elements. 
It is often common to require a reference to derived geometry, such as a center plane of a feature. Such a feature would be modeled through the appropriate subtype of Derived_non_feature_shape_element (a Shape_element that is not on the physical boundary of the part). Figure 6 contains the MIM mapping of the Centre_plane AO for the common case in which the derived center-plane is defined by two faces of the model.

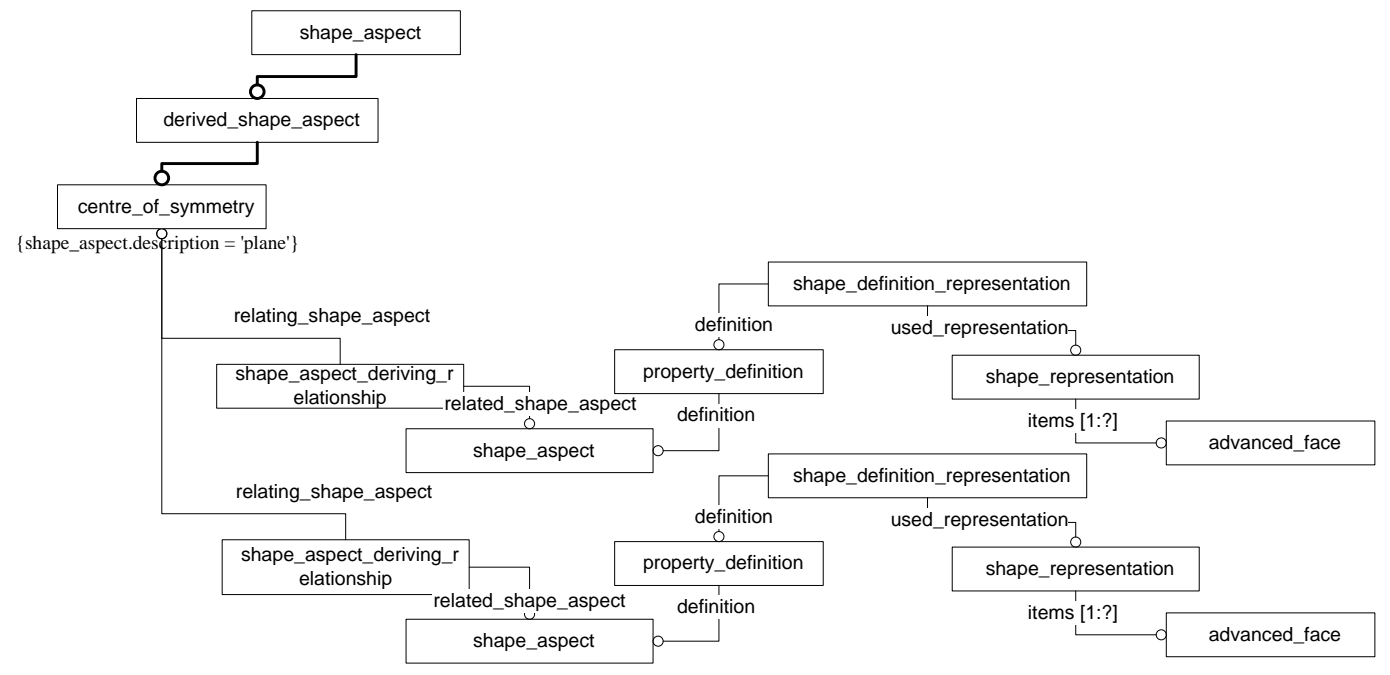

Figure 6. MIM representation of a Centre_plane (a Derived_non_feature_shape_element).

In the context of GD\&T for AP210 package models, it is often necessary to reference surfaces of individual terminals as well as groups of terminals for the purposes of

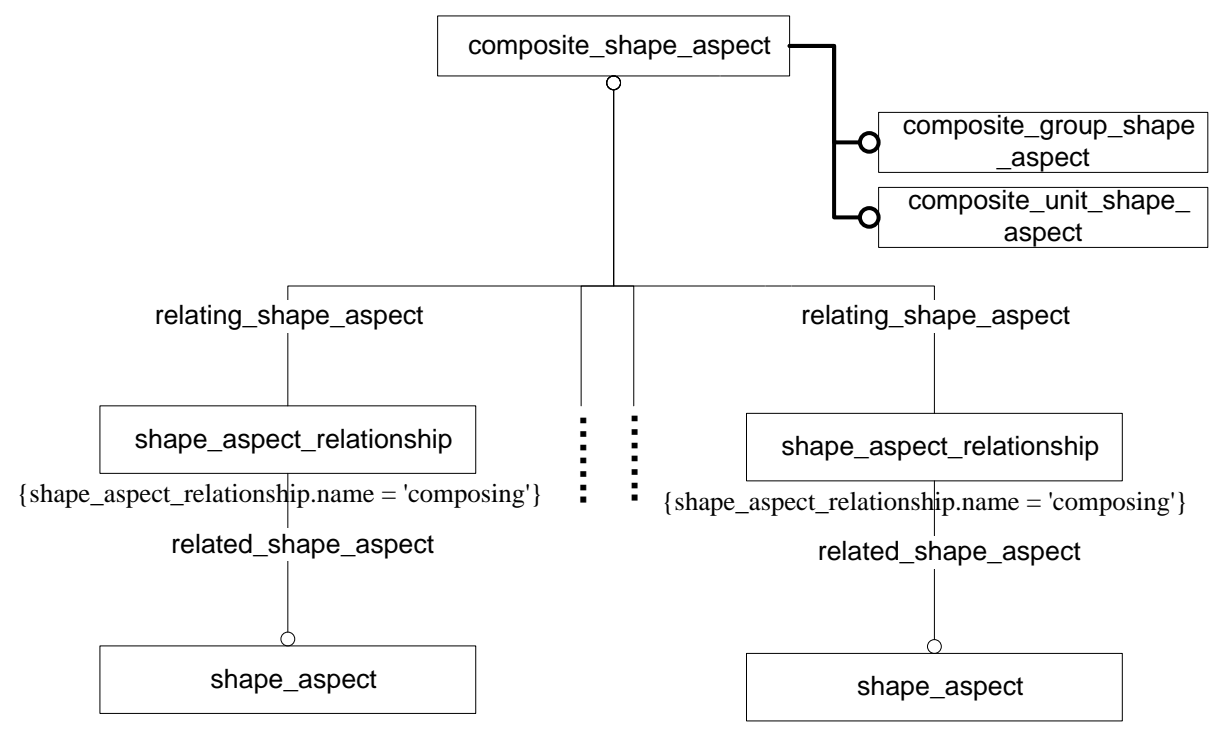

Figure 5. MIM representation of a Composite_shape_element 
defining datum and geometric tolerances. As discussed previously, in an AP210 package model, it will often be necessary to individually reference instances of faces used in a common template (for example, assigning a dimension between the outer-faces of two specific terminals instances). Individual instances of the terminal will typically be placed with respect to the shape model of the package using a usage_concept_usage_relationship. This case is analogous to a common issue in assembly-level tolerance representation. A mechanism for specifying the required association between a shape_aspect and a specific face of an individual terminal instance has recently been established through the MIM entity chain_based_item_identified_reprsentation_usage, a subtype of item_identified_representation_usage, defined in Technical corrigendum 2 to International Standard ISO 10303-41:2005. As the name implies, the new subtype supports a hierarchically linked chain of representations so as to allow unambiguous reference to a representation_item in a lower level of the hierarchy. The MIM mapping for a Shape_element needed to identify an individual face on a individual terminal using the chain_based_item_identified_reprsentation_usage is detailed in Figure 1, where the associated entities, attributes, and relationships are highlighted in red.

\section{Representation of dimensional tolerances}




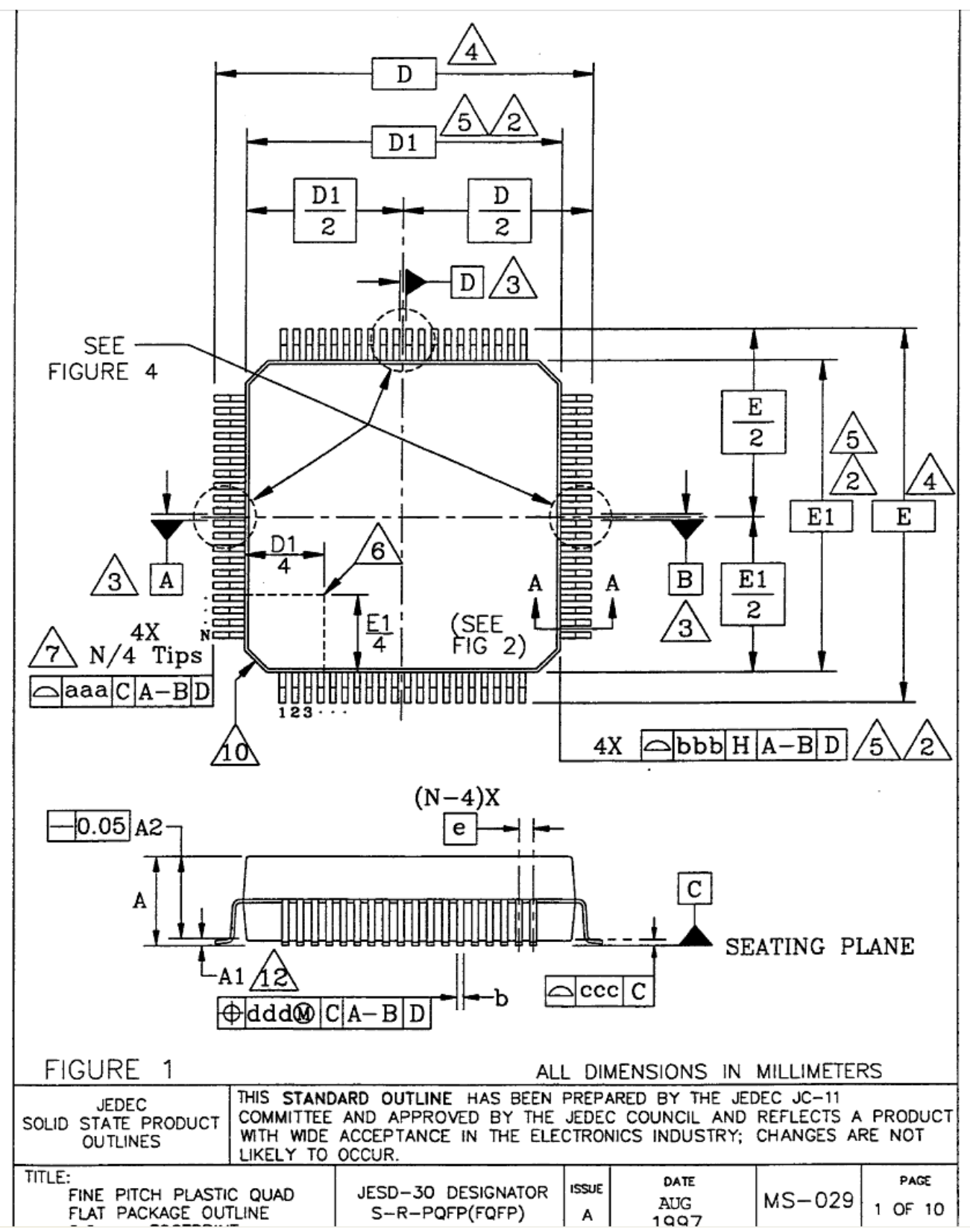

Figure 7. JEDEC Pub. 95 toleranced specification of the QFP package family (MS-029, Figure 1).

The JEDEC Publication 95 standards employ a combination of dimensional tolerances and geometric tolerances. Figure 7 and Figure 8 contains a representative dimensioned and toleranced drawing of a typical JEDEC component package. As may be seen in the figure, there are a number of basic dimensions ( $D, D 1, e$, etc.) as well as a number of dimensional tolerances (A, A1, A2), and 5 geometric tolerances specified in control frames.

The toleranced dimensions are all undirected, and are both dimensional and size tolerances. Dimensions A2 and b are size dimensions (height of the body of the package, 

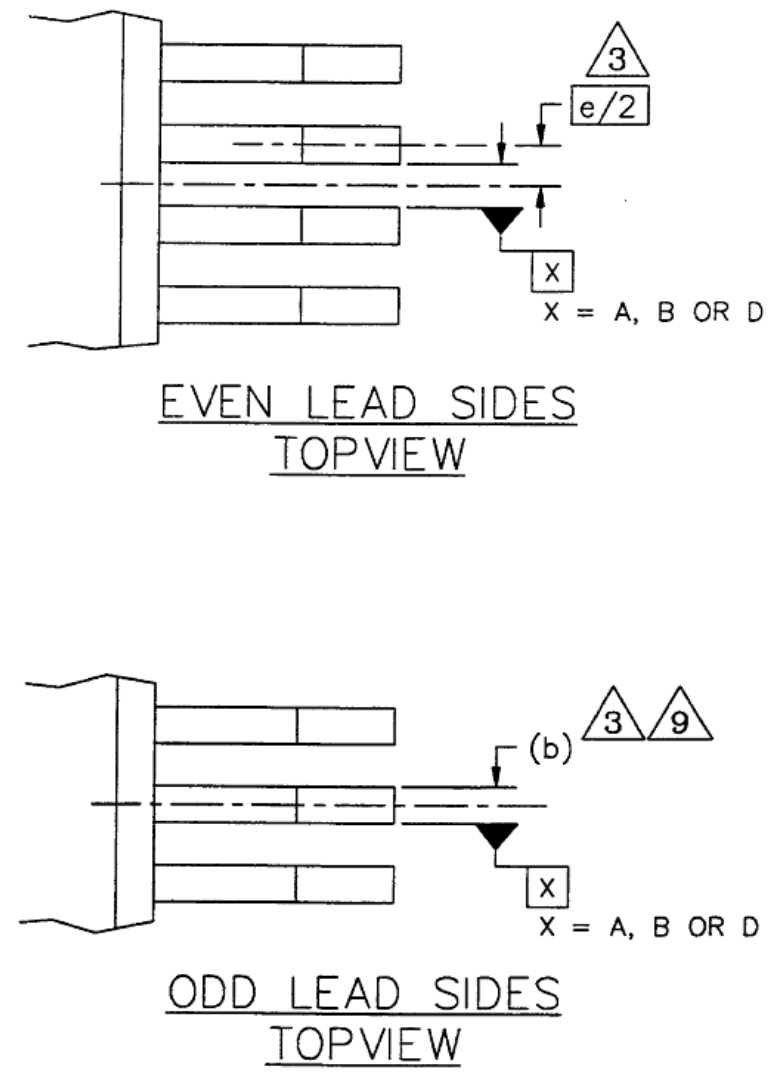

Figure 8. A dimension and datum specified by a feature of size (from JEDEC Pub. 95, MS-029 Figure 4). width of a terminal, respectively). In each case, the size dimension applies to a regular feature of size (two opposed parallel surfaces). In the case of dimension A2, there is a single package body, whereas dimension b must apply to each of the terminals in the package.

The top-level ARM application objects and attributes used in the representation of a dimensional tolerance are contained in Figure 9 and Figure 10. Dimensions A2 and b would be represented as the Height_size_dimension and width_size_dimension subtypes of Size_dimension. Each would refer to a single Shape_element. In the event that the geometric model of the terminal was contained within the package_terminal_template _definition, a single dimension would intrinsically apply to all terminals in the package. In the event that a single solid was used to represent the complete package

model, it would be necessary to use a Composite_group_shape_element which would explicitly contain a shape element for each of the terminal instances in the package. Each of these shape elements would need to reference the two opposing faces (advanced_face) of the terminal within the advanced_brep_shape_representation. For the majority of the JEDEC dimensions, either a minimum / maximum range, or minimum / nominal / maximum values are provided explicitly. The dimensional_value attribute would therefore reference either a Tolerance_range or a Dimension_value_with_limitation. In certain cases, only a minimum or maximum value is provided. In this case, a Value_limit would be used for the representation. The MIM mapping for these ARM concepts for dimensional tolerances are contained in Figure 11 and Figure 12.

Dimensions A and A1 would be represented as instances of the Linear_distance_dimension, subtype of Location_dimension. Neither of these dimensions relates to a feature of size, and would require two distinct Shape_element, each of which would be associated with a particular surface, or group of surfaces in the model. In both cases, the dimension relates the bottom surfaces of the terminals to a face on the body (either top or bottom surface). The bottom surfaces of the terminals could be referenced through a single Shape_element in the case where all terminals are 
instances of the same package_terminal_template_definition. In the event that a single solid was used to represent the entire package, all bottom terminal surfaces should be aggregated together as a Composite_unit_shape_element.

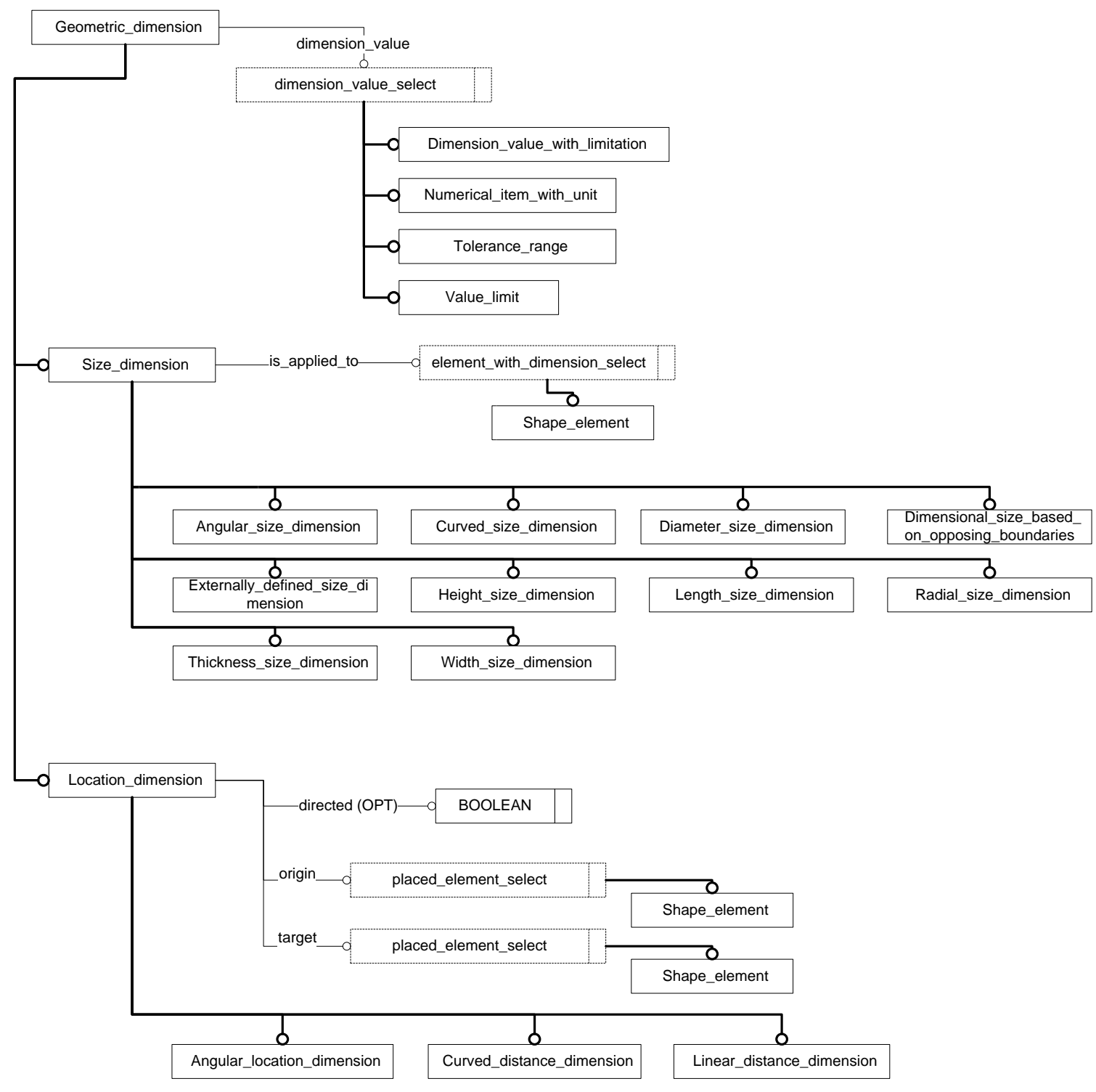

Figure 9. The top-level subtypes and key entities of the Geometric_dimension AO.

\section{Representation of datum}

In this document, we consider three common means of defining a datum plane in the JEDEC Publication 95 specification. Per ASME Y14.5 (1994), a datum is a theoretically exact point, line or plane derived from geometric counterpart(s) on the physical model. The geometric counterpart is known as a datum feature. The AP210 ARM supports a family of application objects (AOs) that are subtypes of Datum. Several of the commonly used subtypes that are most relevant for the domain of packaged electronic components are documented in Figure 13. 


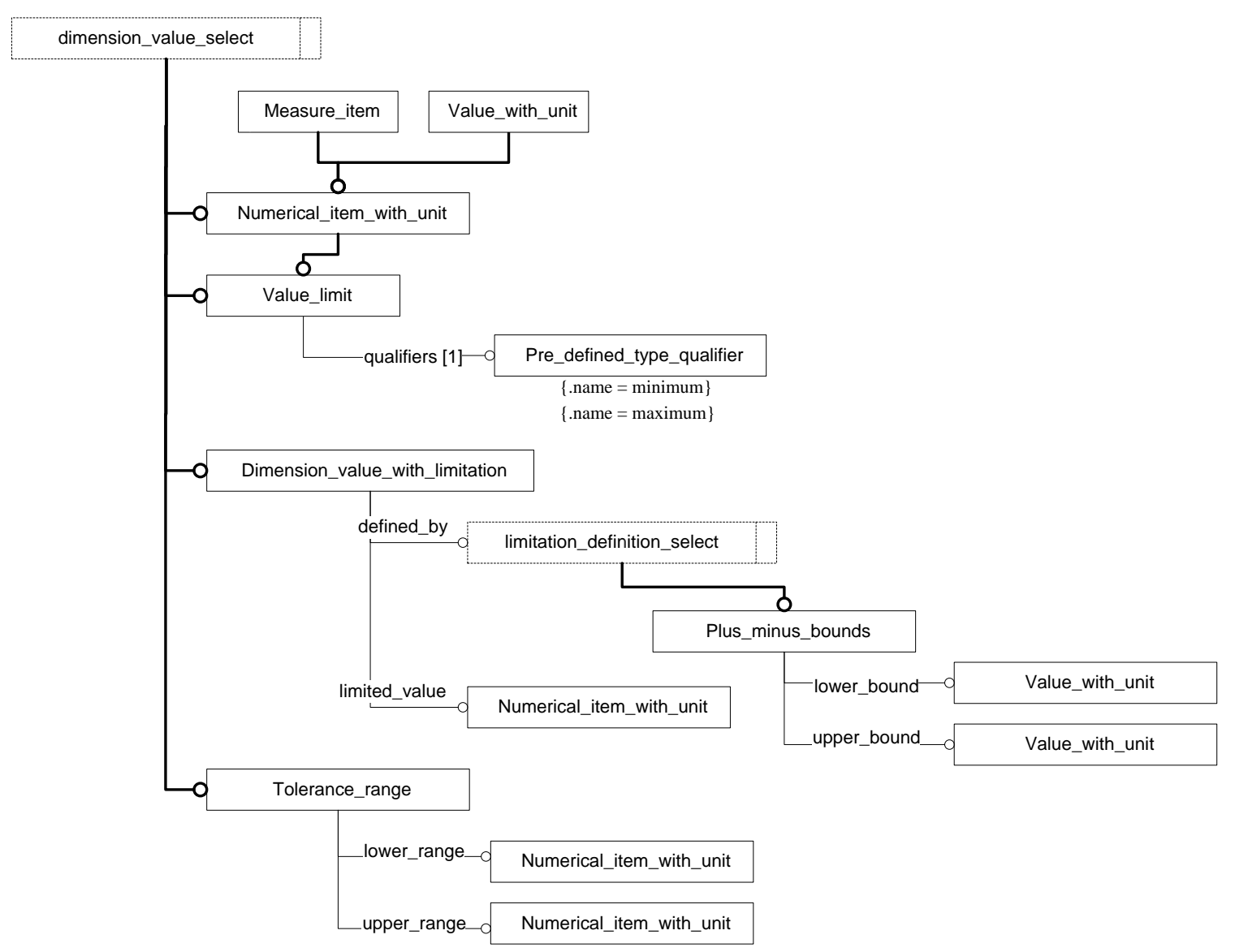

Figure 10. ARM AOs for expressing a value of a Geometric_dimension.

In many cases, a datum feature will correspond to either a surface feature of a part or a "feature of size" of a part. When it is required that a specific region of a surface be used to determine the datum, a datum target may be used. A "feature of size" must contain opposed points, and have a reproducible derived median point, axis, or center plane. An example of an internal feature of size would be a slot. A pin or a shaft would be an example of an external feature of size. Many times, a feature of size is used as a datum feature in defining a datum.

A common example in the context of JEDEC Publication 95 would be the use of a package terminal as a feature of size to define a datum plane. Figure 8 outlines how either an internal or an external feature of size would be called out as a datum feature, depending on whether a particular package had an even or odd number of leads on a particular side. In the case of an odd number of leads per side, an external feature of size may be associated with the opposing sides of the lead exiting the body of the component at its center plane. In the case of an even number of leads per side, an internal feature of size may be associated with the opposing (inner) sides of the two center-most leads.

The boxed $\mathrm{x}$, in conjunction with the filled triangle constitutes the datum feature symbol [Y14.5-1994 3.3.2]. In the example of Figure 8, datum features A, B, and D are all defined equivalently. 
Datum features A, B, and D represent a derived datum center plane.

\begin{tabular}{l|l|}
\hline \multicolumn{1}{c|}{ dimensional_size } & \\
\cline { 2 - 2 } \{dimensional_size.name $=$ 'thickness size'\} \\
\{dimensional_size.name $=$ 'width'\} \\
\{dimensional_size.name $=$ 'length'\} \\
\{dimensional_size.name $=$ 'height'
\end{tabular}
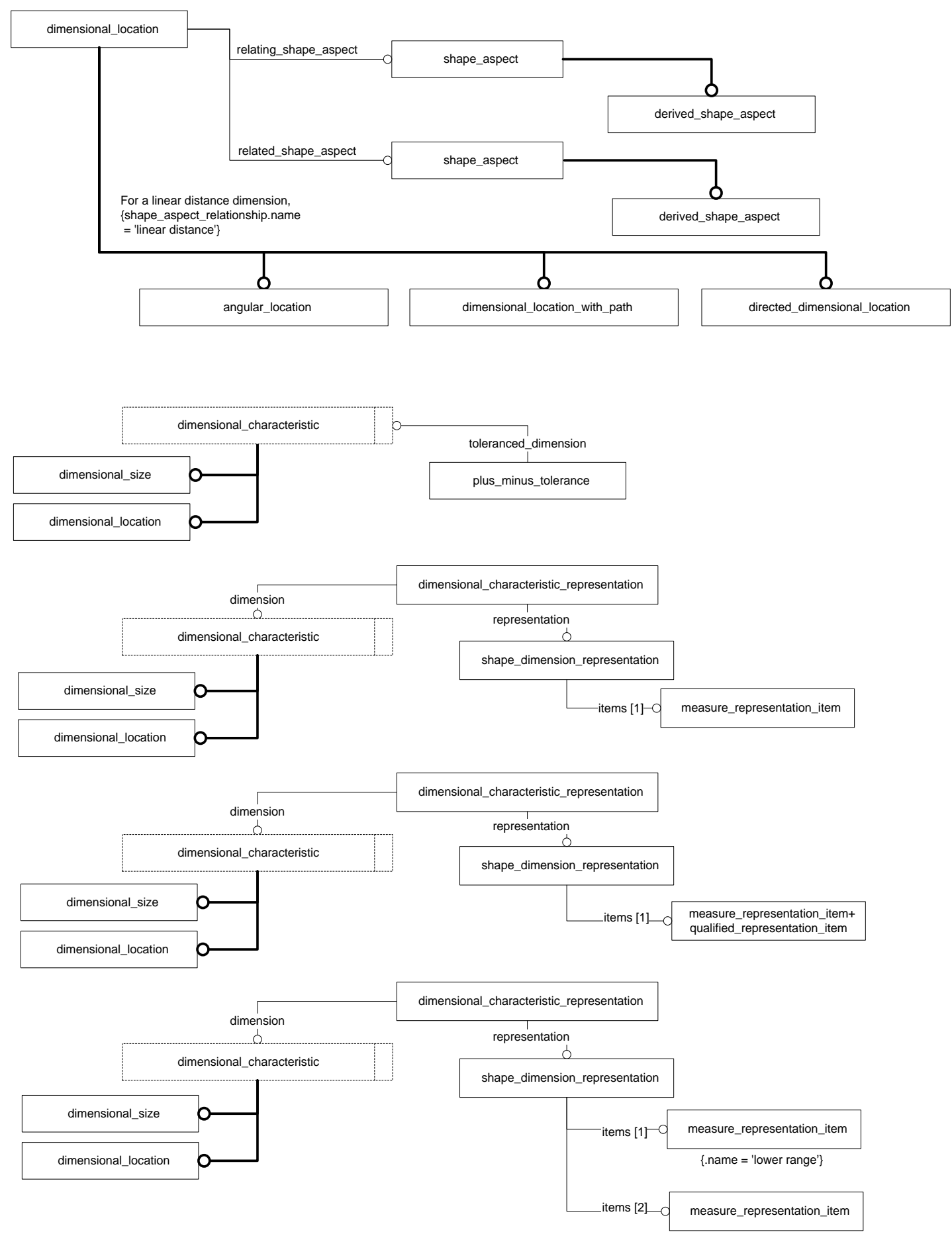

Figure 11. MIM mappings of the Geometric_dimension AO. 


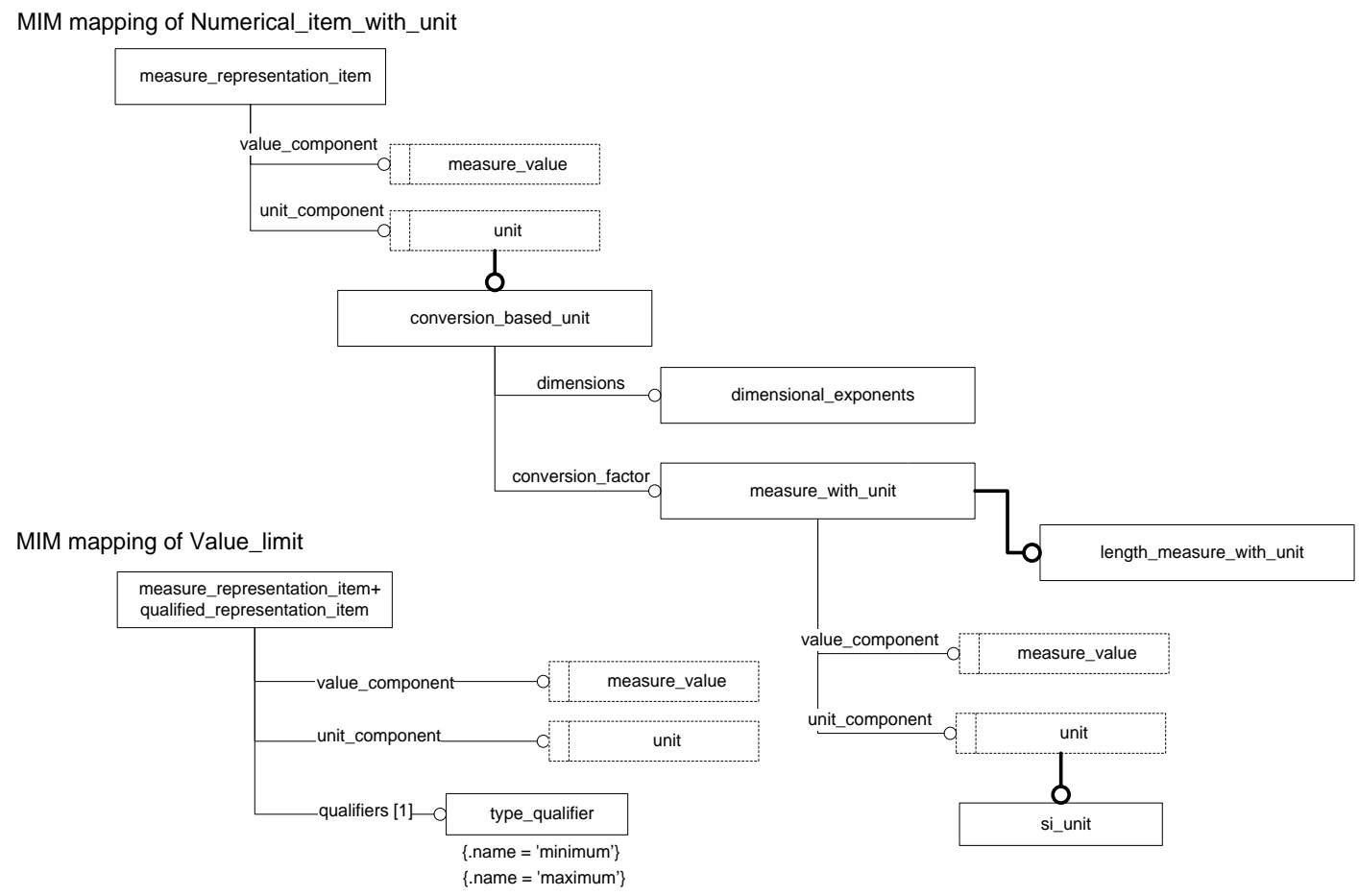

MIM mapping of Dimension_value_with_limitation

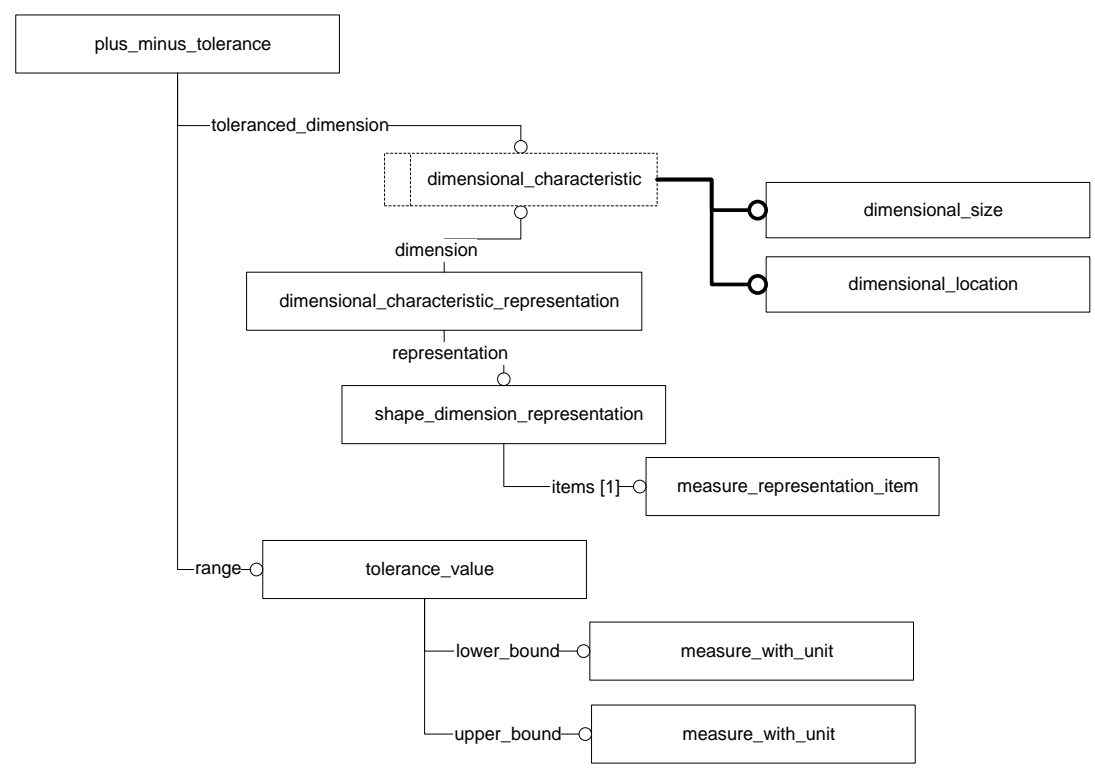

MIM mapping of Tolerance_range

shape_dimension_representation

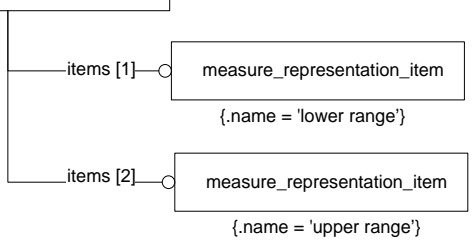

Figure 12. MIM mappings of the alternate representations for a dimension value. 


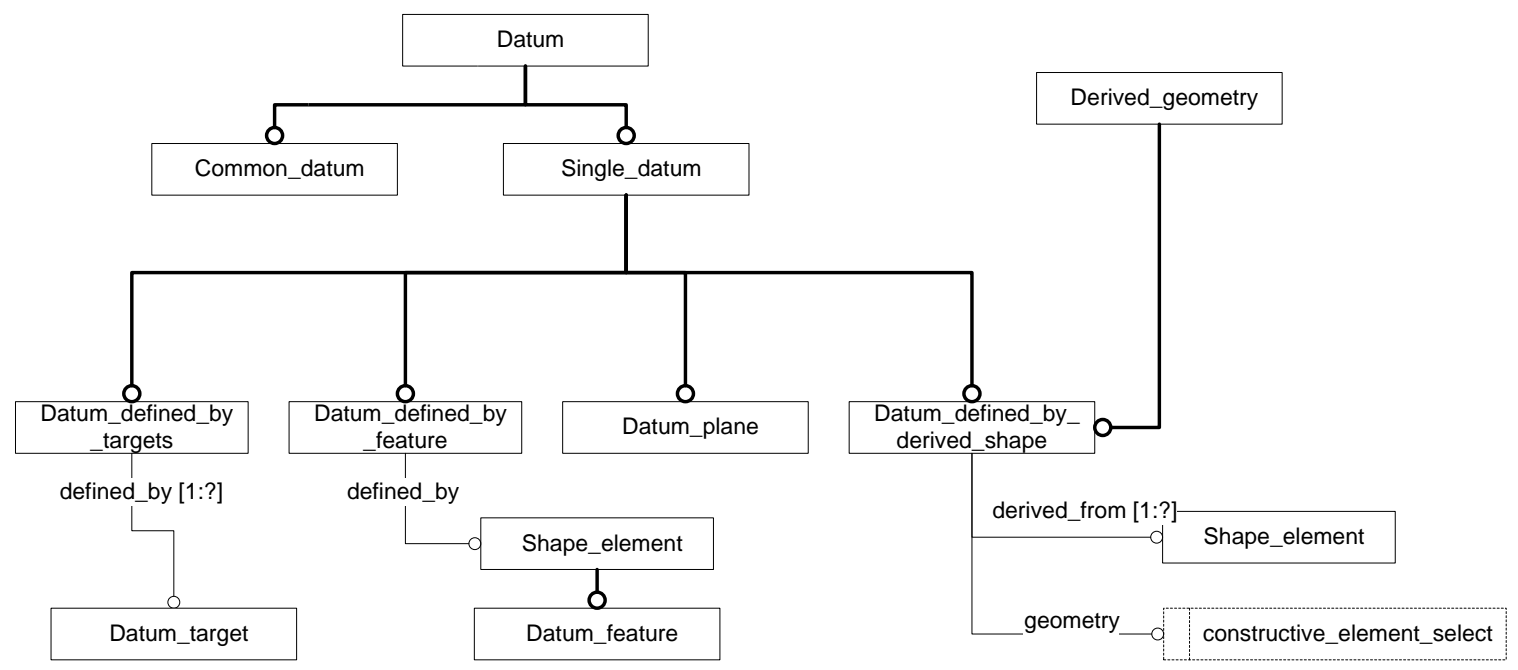

Figure 13. Selected subtypes of the Datum application object

The suggested recommended practice is to use the ARM application object Datum_defined_by_derived_shape where the derived shape is a Centre_plane. The center plane must reference the two surfaces of the internal or external datum feature. These two surfaces are a Shape_element of the model. The relationships between these AOs are depicted in Figure 14 below. The use of the complex entity Datum_defined_by_derived_shape+Datum_plane conveys the information that this is a datum plane as well as a datum defined by derived geometry (i.e. a center plane).

The MIM mapping of the AOs in Figure 14 is contained in Figure 15. The Datum_defined_by_derived_shape maps to the datum entity, and the Centre_plane maps to the centre_of_symmetry entity, both subtypes of shape_aspect. In both cases, the inherited attribute shape_aspect.description

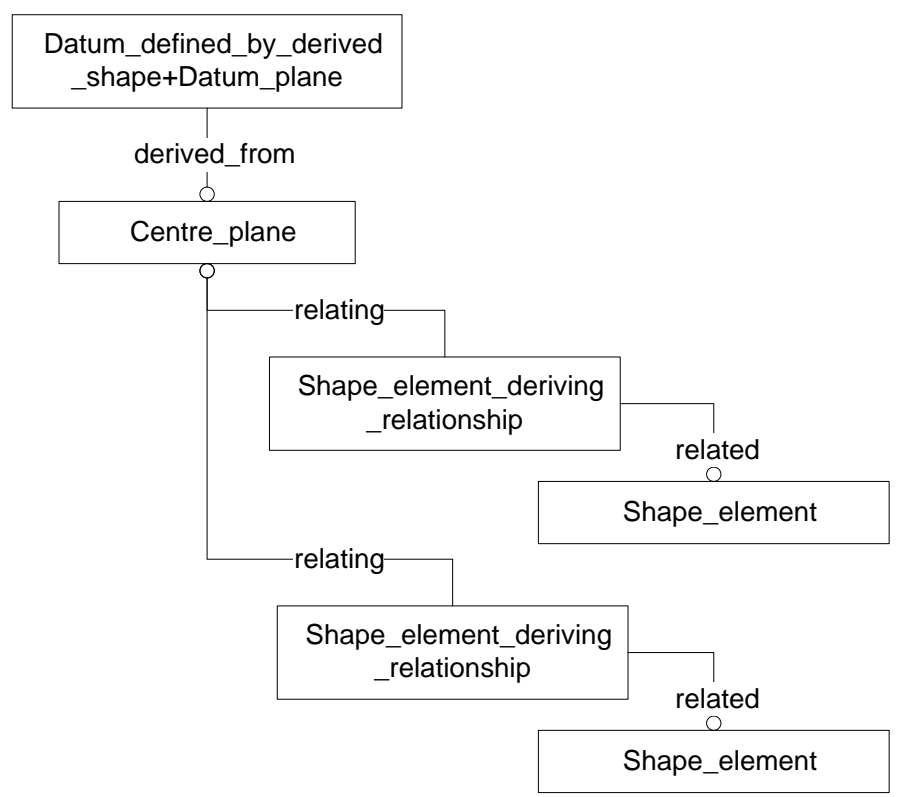
is set to the value 'plane' in order to appropriately specialize the two entities. As may be seen in Figure 15, the centre_of_symmetry is defined by two entities of type shape_aspect, each of which has an advanced_face as its shape_representation.

There are four datum planes defined in the JEDEC Pub. 95 specification of the QFP package family (see Figure 7). Datum features A, B, C, and D are used to define four datum planes, A-B, C, and D which

Figure 14. A datum defined by a derived center plane. 


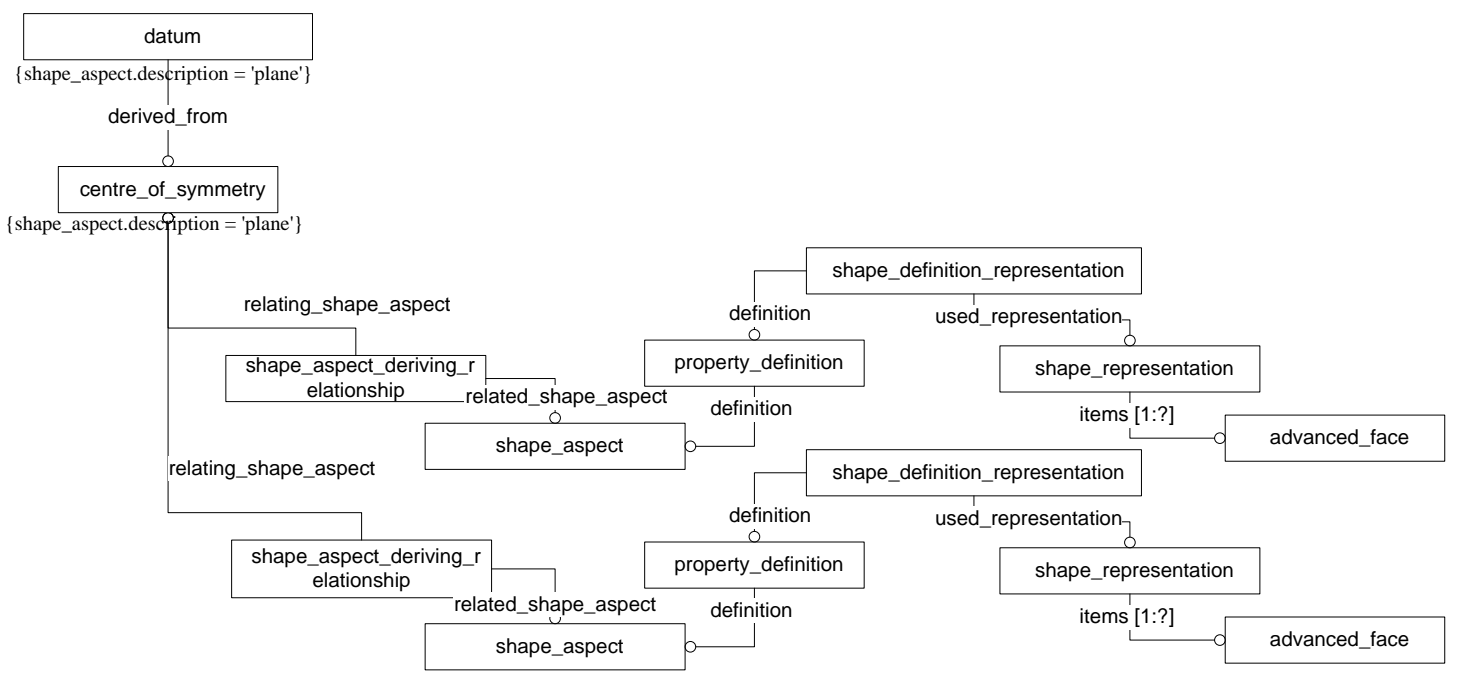

Figure 15. MIM mapping of a derived center plane (and a datum defined by derived center plane).

together constitute a datum reference frame.

Datum features A, B, and D would be modeled as discussed previously (see Figure 14 and Figure 15). Datum A-B would be modeled as a common datum (composed of datums $\mathrm{A}$ and $\mathrm{B})$.

The ARM AO Common_datum is composed of a minimum of two Single_datum AOs. Both Datum_plane and Datum_defined_by_derived_shape are subtypes of Single_datum (see Figure 13). The MIM mapping for the A-B Common_datum AO and its relationship with the two individual datum planes is detailed in Figure 16. 
Datum C represents the seating plane. As specified in Design Guide 4.4a of JEDEC Pub. 95, the seating plane is the "plane established by three or more leads that support the device when it is placed on top of a horizontal planar surface.... The feet of the leads are datum features that ... establish the datum plane.”

In order to associate a single datum plane with the feet of the leads as datum features, a complex instance of the ARM AOs composite_unit_shape_element and Datum_feature may be employed, as it is intended that the collection of all of the lead feet be treated as a single unit, as opposed to a group (see also Composite_group_shape_element). If choosing to model the collection of datum features through a Composite_unit_shape_element, the datum itself can be modeled as a Datum_defined_by_feature+Datum_plane. The top-level ARM AOs and corresponding MIM mapping for the seating plane datum are detailed in Figure 17.
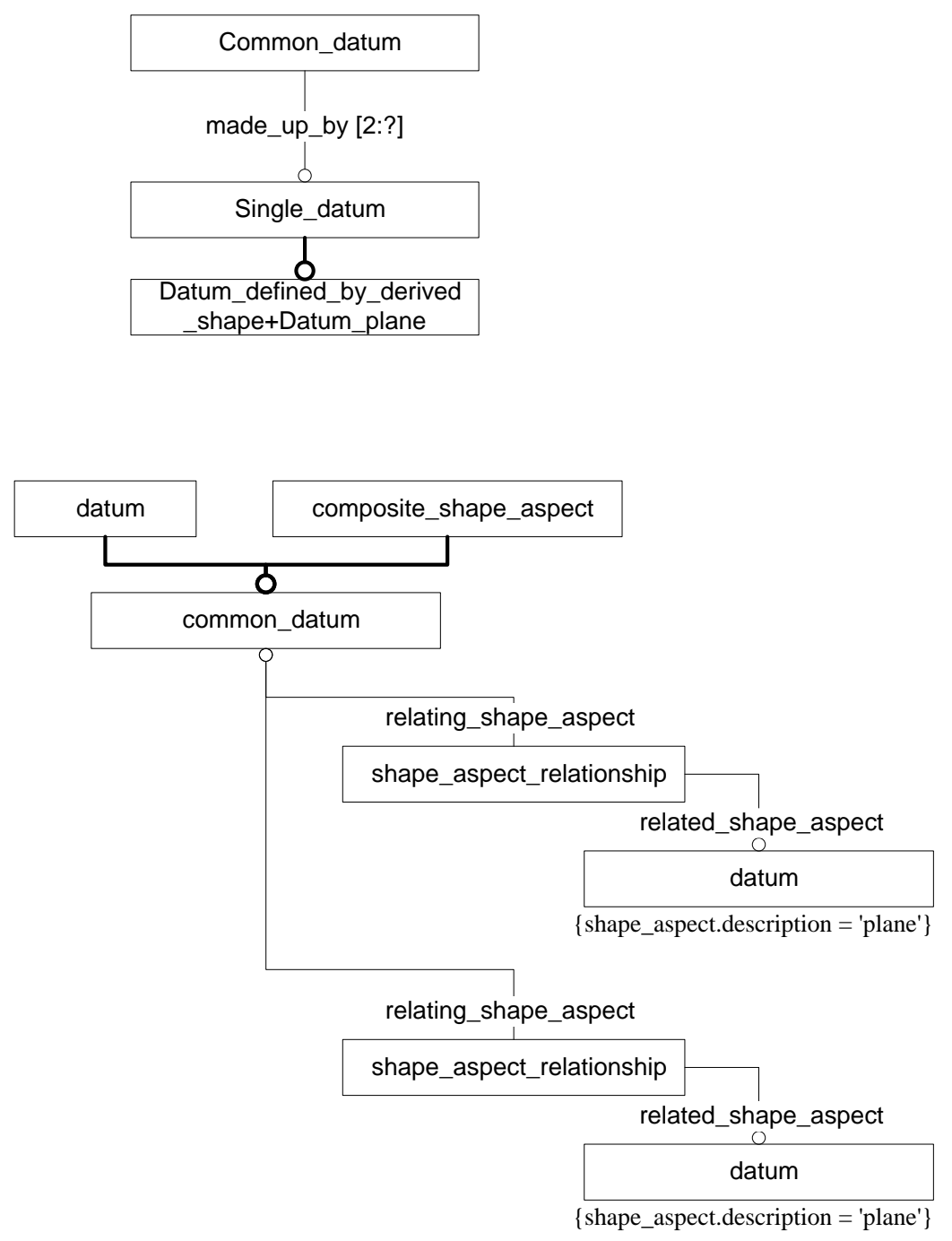

Figure 16. The ARM Common_datum AO and MIM mapping of the A-B datum plane. 

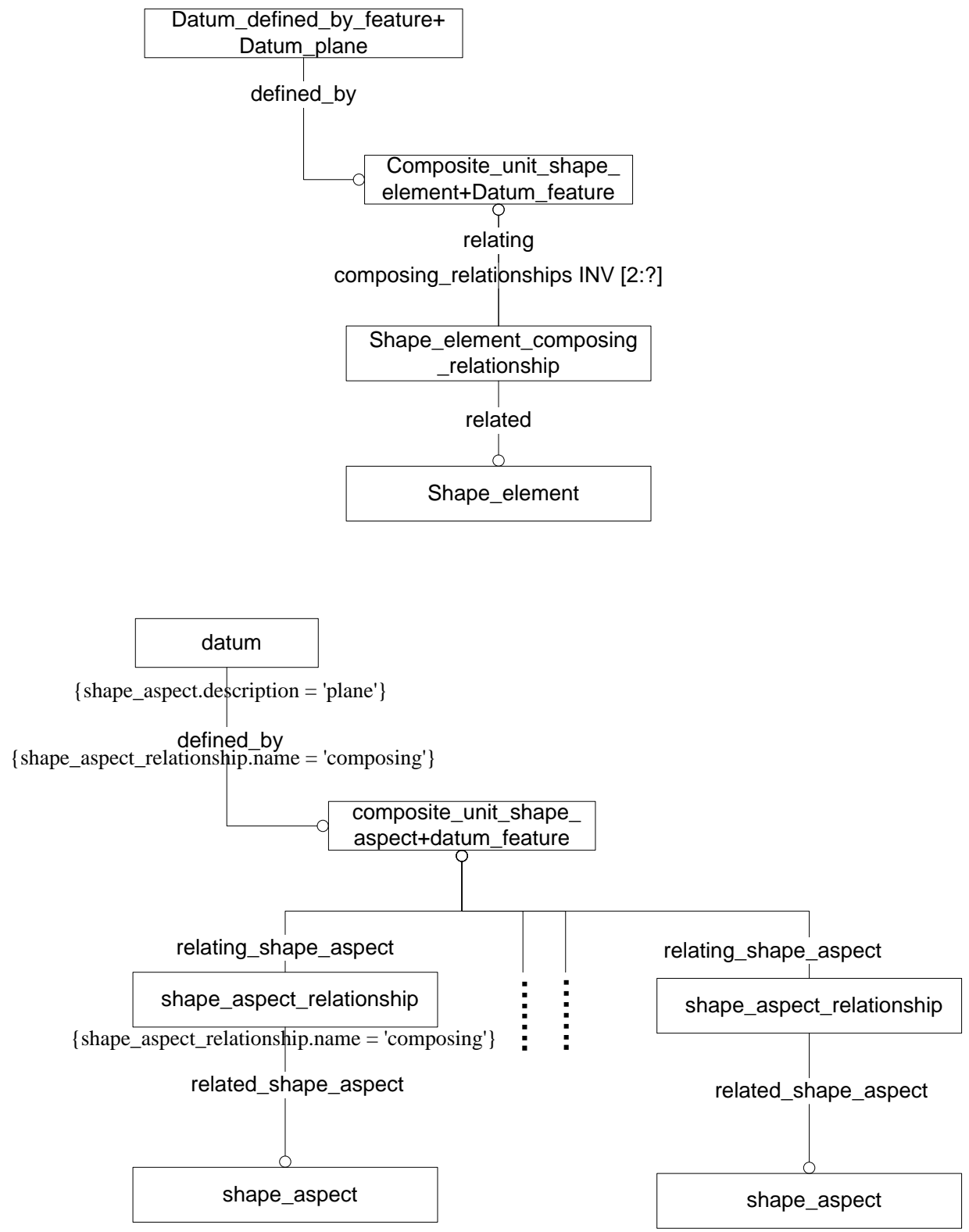

Figure 17. The top-level ARM AOs to be used in the representation of the seating plane (datum C) and their corresponding MIM mappings.

\section{Representation of Geometric Tolerances}

In addition to several dimensional tolerances, a number of geometric tolerances are contained in Figure 7. The top-level ARM application objects used to represent geometric tolerances are contained in Figure 18. Figure 19 contains the MIM mapping for the geometric_tolerance hierarchy, as well as a number of the key attributes. Tolerances aaa, bbb, and ccc all represent surface profile tolerances. All three have datum references (optional for a surface profile tolerance), and none contain a material condition modifier. For this reason, each would be represented as a complex instance of geometric_tolerance_with_datum_reference+surface_profile_toleranc 


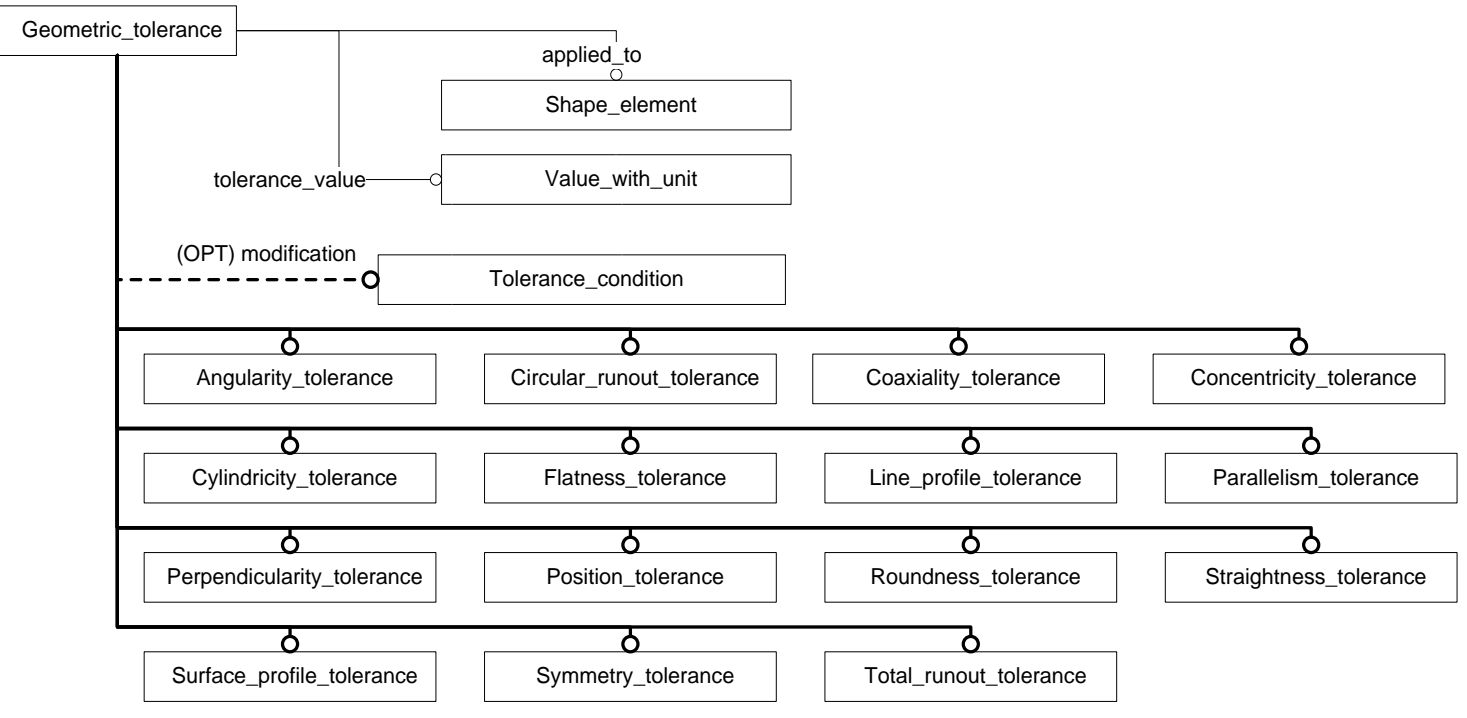

Figure 18. The top-level subtypes of the Geometric_tolerance AO.

e (see Figure 20). The magnitude of the tolerance would be represented as a length_measure_with_unit. In order to communicate the datum references, and their sequence within the datum reference frame, the geometric_tolerance_with_datum_reference.datum_system attribute will contain an aggregate of datum_reference. As shown in Figure 20, each of the datum_reference entities contains an integral precedence value, as well as a reference to an individual datum. Finally, the tolerance must reference a shape_aspect. In the case of surface profile tolerance bbb, there are four instances of the package body side surface that are controlled by the tolerance. These four surfaces (each represented with a shape_aspect associated with an advanced_face) would be grouped together through a composite_group_shape_element. In the case of tolerance aaa, either a single shape_aspect, or an aggregation of shape_aspect will be used, depending on the representation of the underlying package model.

Positional tolerance ddd is the only positional tolerance called out in Figure 7. This tolerance has a maximum material condition (MMC) modifier, in addition to the datum references. A positional tolerance only controls position of features of size. In this case, tolerance ddd controls the positional accuracy of the center plane of the package terminals. When the MMC condition is applied to modify the location tolerance of a size feature, the tolerance applies at the maximum material condition of the feature. If a material condition modifier is not present, RFS (regardless of feature size) applies automatically. 
For integral features, shape aspects.product definitional=".TRUE."

For derived elements shape aspects.product definitional=".FALSE."

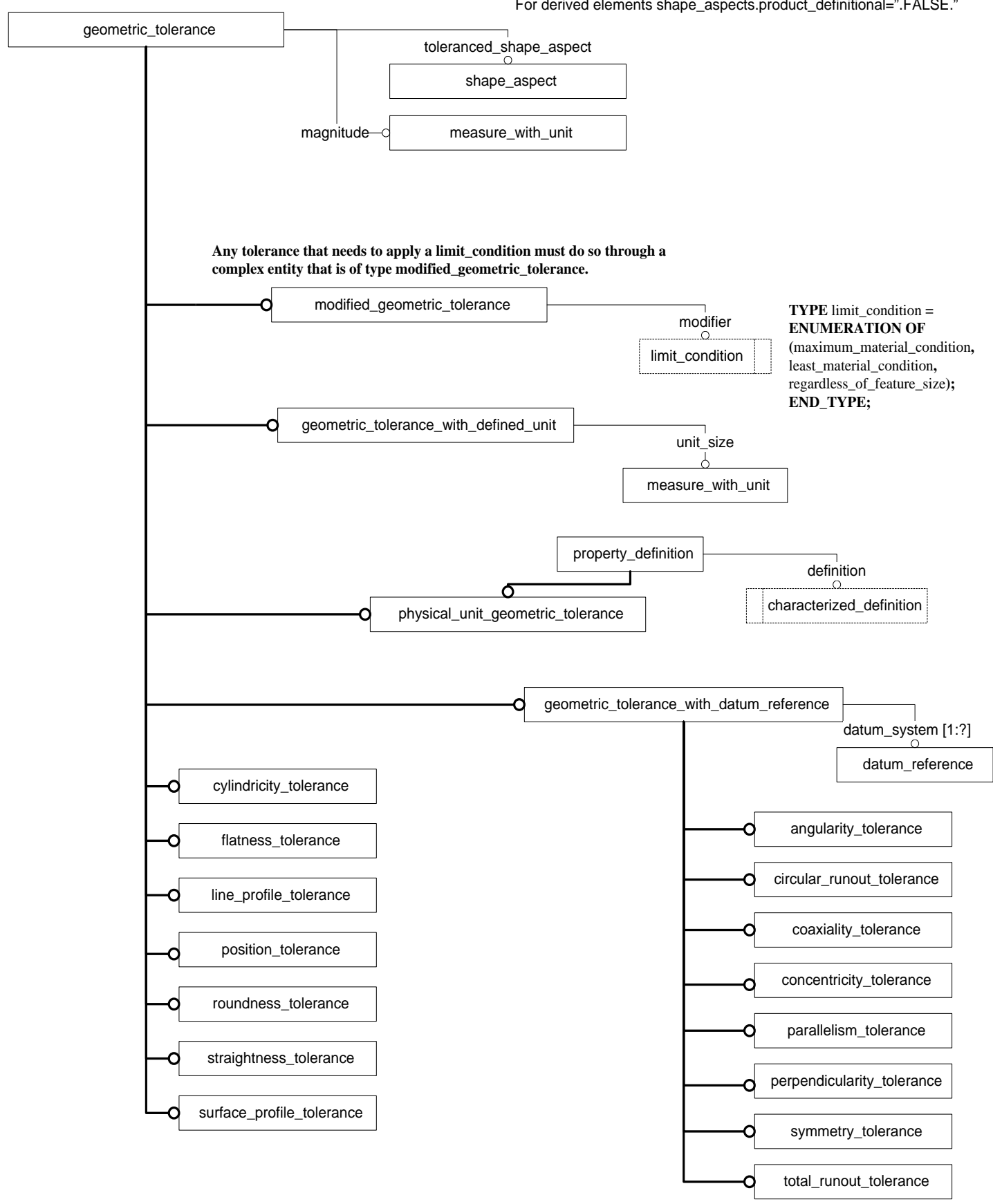

Figure 19. The geometric_tolerance entity hierarchy and certain key attributes.

The two opposing parallel side faces of the terminal constitute an external feature of size. This shape_aspect will always be shared between the positional tolerance and a size dimension (the terminal width b). To represent tolerance ddd, we require a complex of position_tolerance+modified_geometric_tolerance+geometric_toleran ce_with_datum_reference as detailed in Figure 21. 


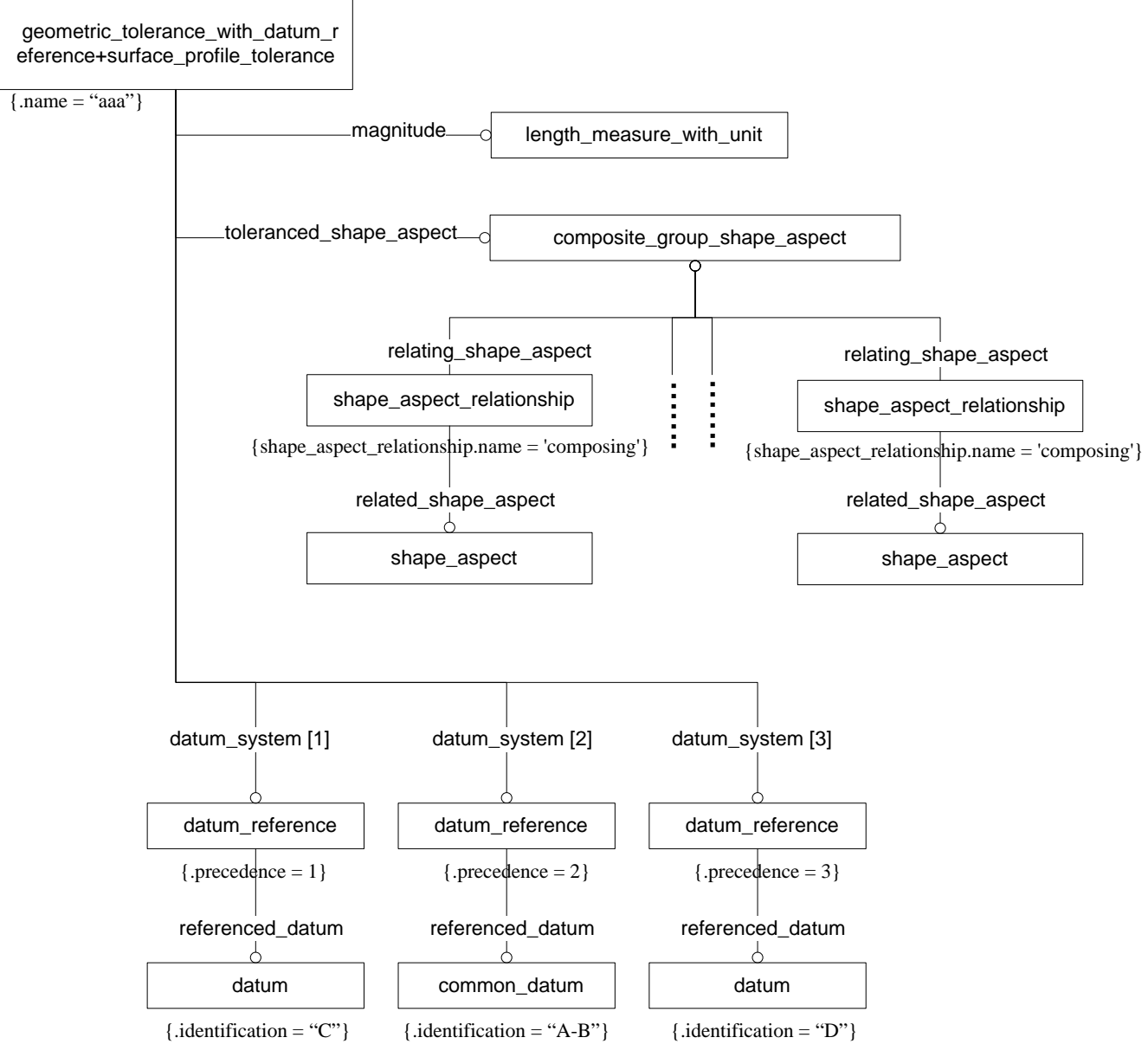

Figure 20. MIM mappings of surface profile tolerance aaa (bbb and ccc similar). 


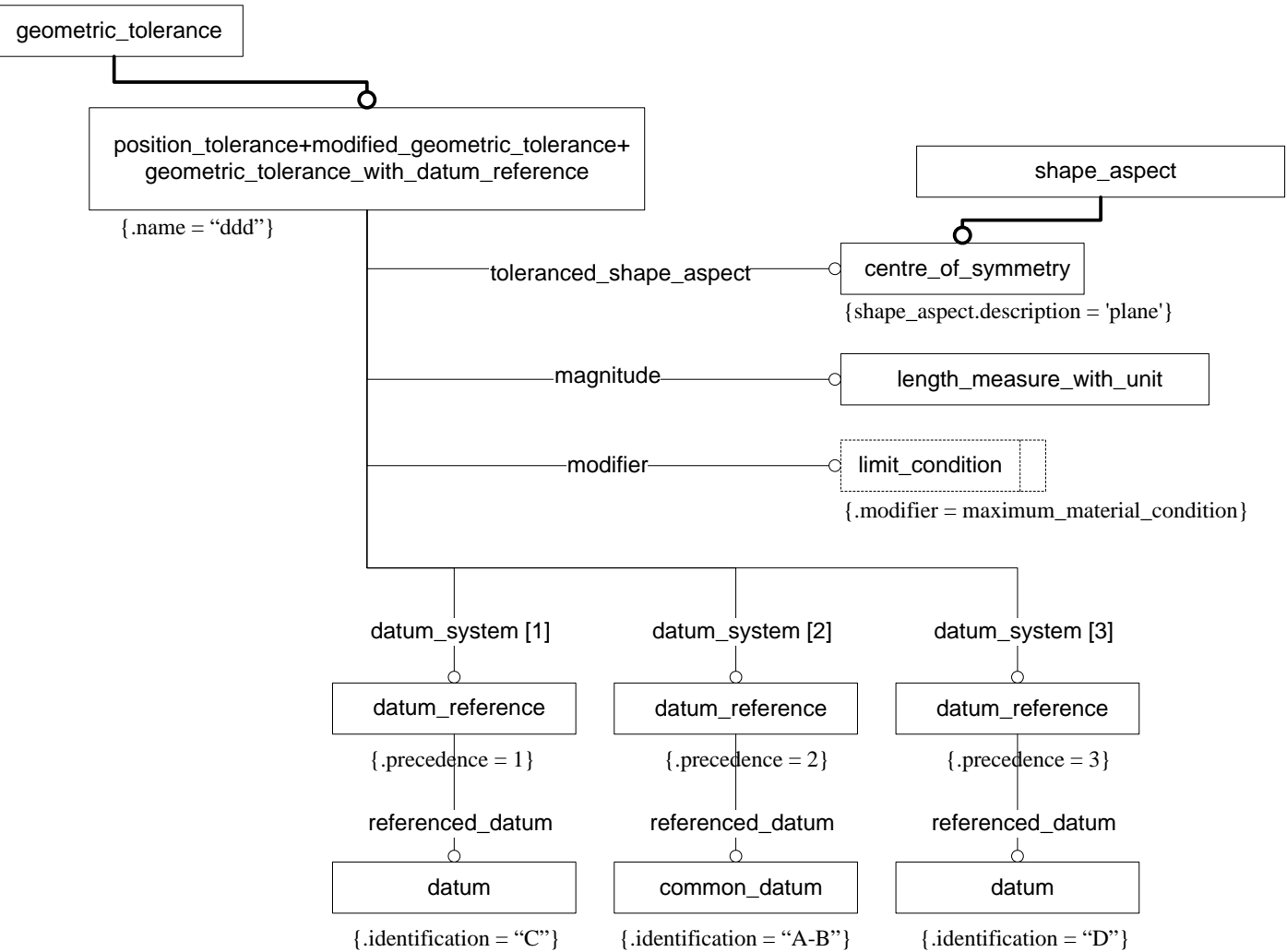

Figure 21. MIM mapping of tolerance ddd. 